Supporting Information for :

\title{
A New Generation of Gold Nanoshell Coated Esophageal Stent: Preparation and Biomedical Applications
}

Jibin Song, ${ }^{\dagger}$ Hao Hu, ${ }^{*}$ Chao Jian, ${ }^{*}$ Kaichun Wu, Xiaoyuan Chen ${ }^{*}{ }^{\dagger}$

${ }^{\dagger}$ Laboratory of Molecular Imaging and Nanomedicine (LOMIN), National Institute of Biomedical Imaging and Bioengineering (NIBIB), National Institutes of Health, Bethesda, Maryland 20892, United States

${ }^{*}$ Sate Key Laboratory of Cancer Biology \& Xijing Hospital of Digestive Diseases, Fourth Military Medical University, Xi'an, China

\section{Corresponding Author}

*E-mail: shawn.chen@,nih.gov. 


\section{Experimental Section}

\section{In Vitro Cytotoxicity of Gold Nanoshell Coated Stent}

A standard Cell Counting Kit-8 (CCK-8) was utilized to analyze the cytotoxicity of gold nanoshell coated stent following a general protocol. Briefly, U87MG and OVARC- 8 cancer cells were seeded in a 96-well plate with the concentration of $1 \times 10^{4}$ cells/well, respectively. After incubation at $37{ }^{\circ} \mathrm{C}$ for $24 \mathrm{~h}, 1-5$ gold nanoshell coated stent wires were placed on the well for $4,10,20,35$ and $50 \mathrm{~h}$, respectively, after which $10 \mu \mathrm{l}$ of CCK-8 solution was added to each well of the 96-well plate and incubated for another $4 \mathrm{~h}$. The amount of an orange formazan dye, produced by the reduction of WST-8 (active gradient in CCK-8) by dehydrogenases in live cells, is directly proportional to the quantity of live cells in the well. Therefore, by measuring the absorbance of each well at $450 \mathrm{~nm}$ using a microplate reader, cell viability could be determined with the calculation of the ratio of absorbance of experimental well to that of the cell control well. All experiments were triplicated and results were averaged. 


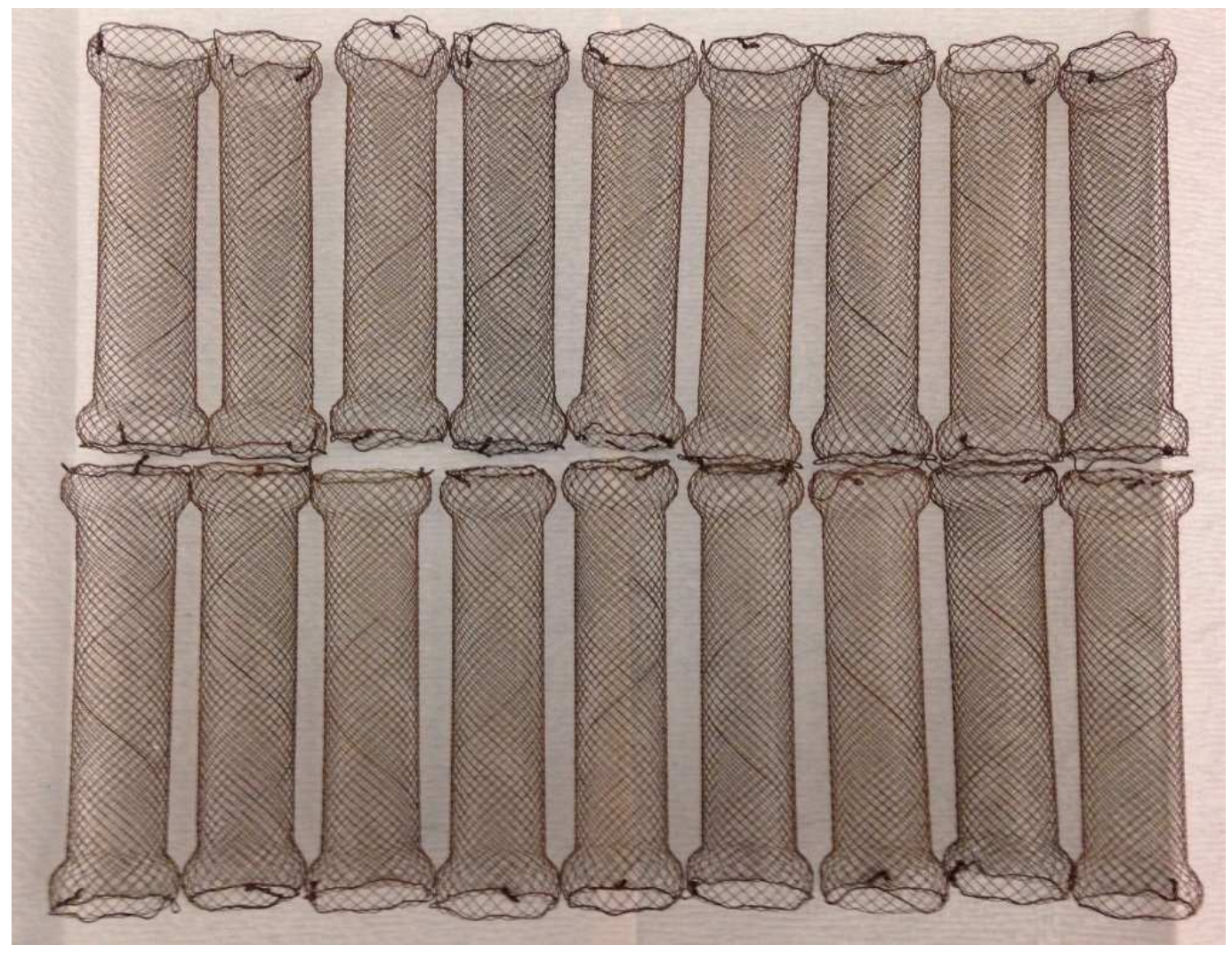

Figure S1. Photographs of the as-prepared eighteen gold nanoshell coated esophageal stens. 


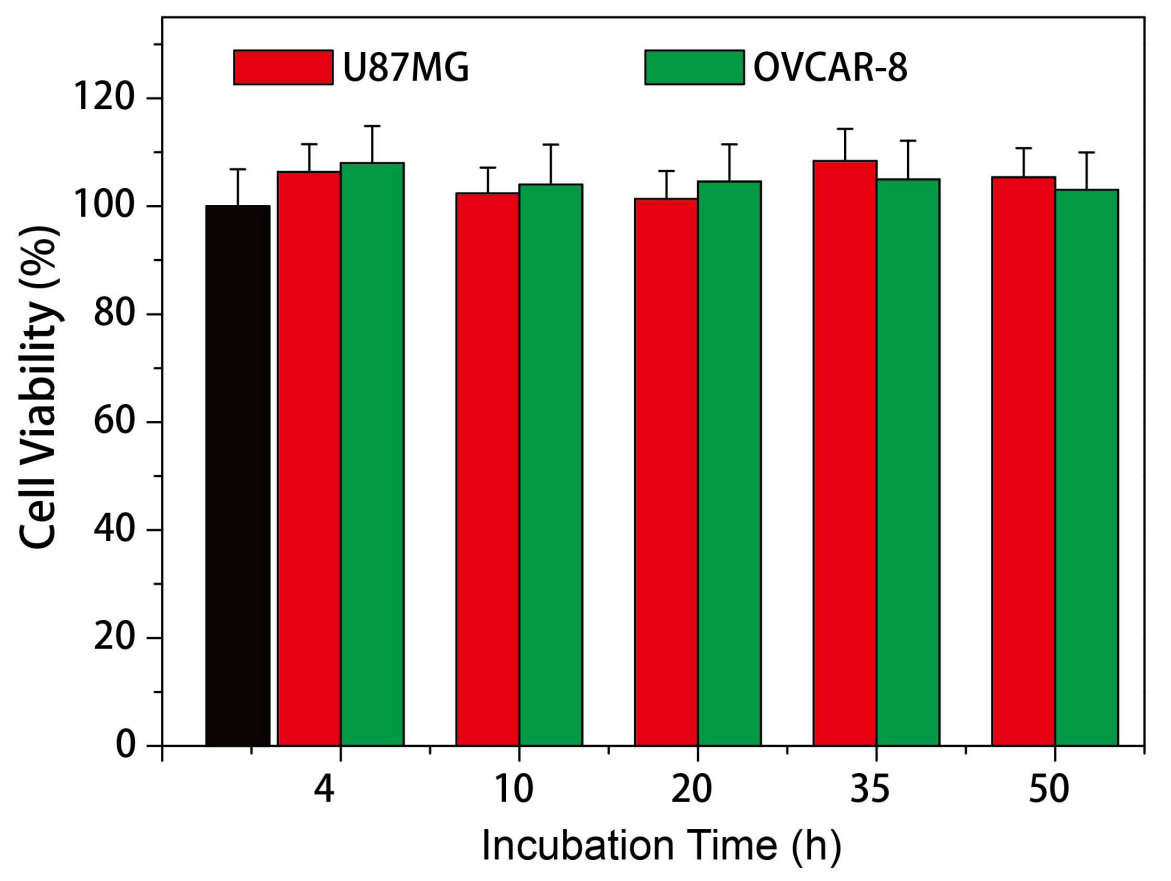

Figure S2. Cytotoxicity of gold nanoshell coated stent after incubation with U87MG and OVCAR-8 cells for different periods of time. 


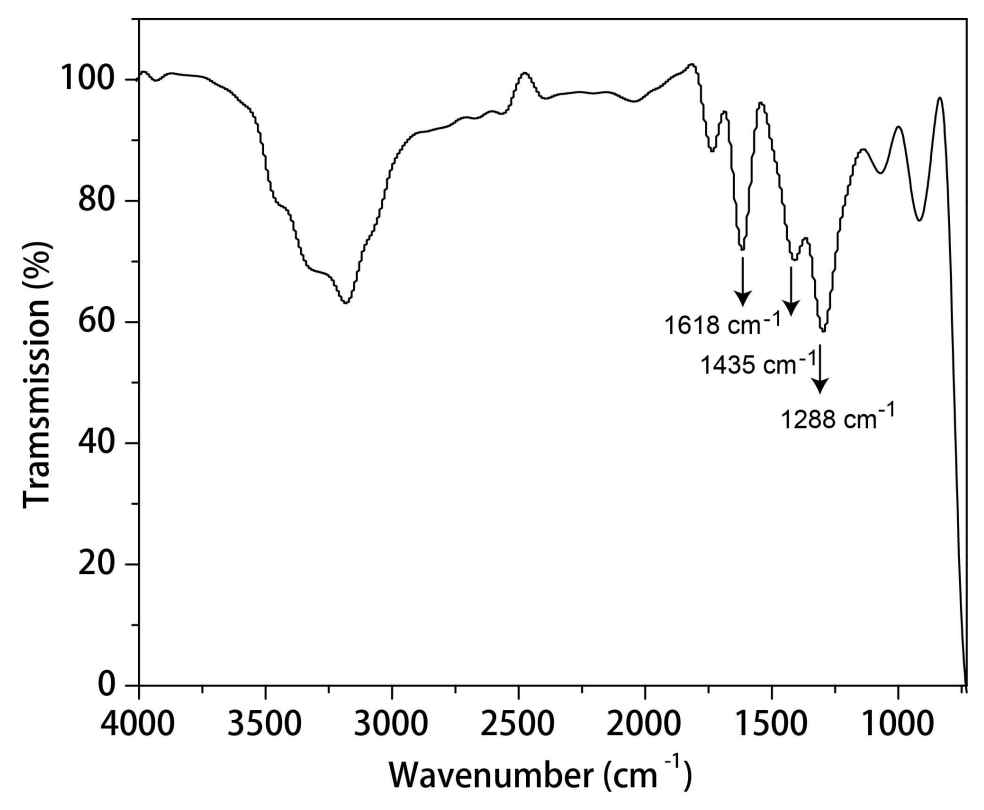

Figure S3. FTIR spectra of PDA coated stent. The presence of PDA on the stent is verified by the appearance of the absorbance peak at $1618 \mathrm{~cm}^{-1}$ (superposition of $\mathrm{NH}$ bending and phenylic $\mathrm{C}=\mathrm{C}$ stretching), $1435 \mathrm{~cm}^{-1}\left(\mathrm{~N}-\mathrm{H}\right.$ scissoring) and $1288 \mathrm{~cm}^{-1}$ (phenoxy C-O stretching).

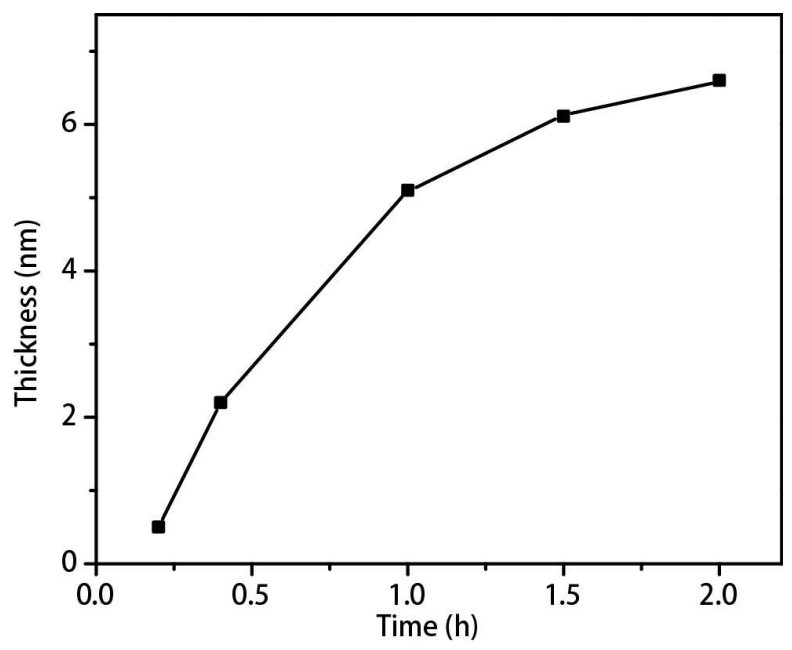

Figure S4. The gold nanoshell thickness increases with increasing reaction time based on the ICP test result. 


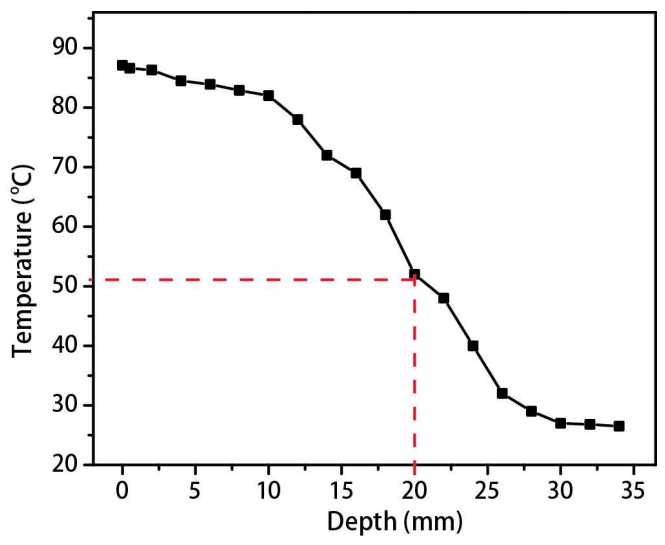

Figure S5. The temperature of the pork at different depths after treatment with AuNS coated stent wire and $808 \mathrm{~nm}$ laser irradiation $\left(0.25 \mathrm{~W} / \mathrm{cm}^{2}\right)$ for $5 \mathrm{~min}$.

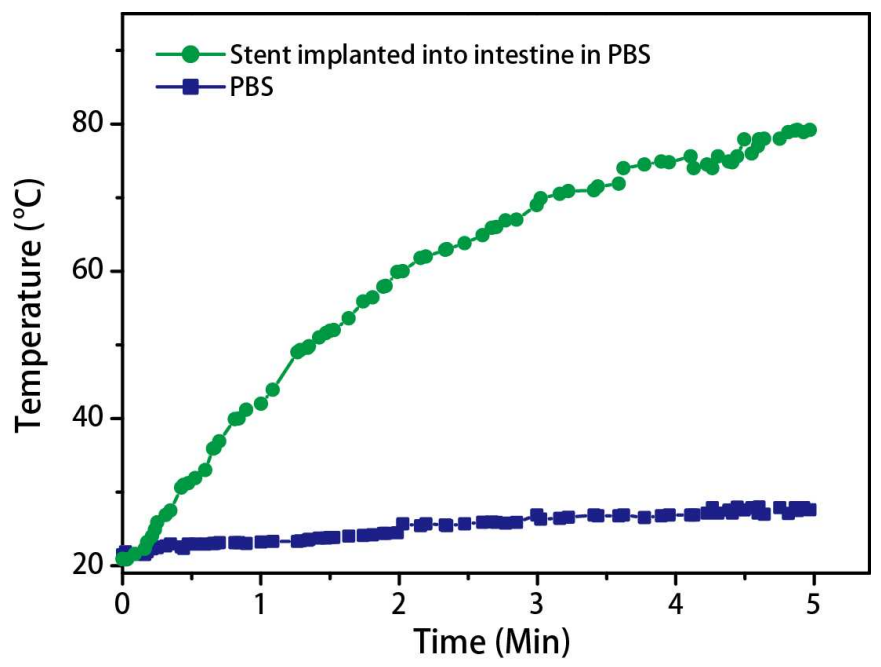

Figure S6. Temperature increase of a stent implanted in intestine and immersed in PBS after irradiation with $808 \mathrm{~nm}$ laser for $5 \mathrm{~min}$. 